\title{
Métodos para a remoção de redundâncias de árvores de falhas
}

\author{
Paulo Renato Alves Firmino (UFPE) praf62@yahoo.com \\ Pedro Igor Carvalho Moreira (UFPE) pedroigor@click21.com.br \\ Rohgi Toshio Meneses Chikushi (UFPE) rohgi toshio@yahoo.com.br \\ Enrique López Droguett (UFPE) ealopez@ufpe.br
}

\begin{abstract}
Resumo
A análise de árvores de falhas é uma importante ferramenta de apoio às inferências quanto à confiabilidade que, por sua vez, é uma das bases da engenharia de produção. O método de BDDs (Diagramas de Decisão Binária) tem sido citado como uma alternativa às técnicas convencionais, que alia tanto maior precisão quanto menor esforço computacional. O grande problema para a aplicação de BDDs reside na necessidade de conversão da árvore de falhas para o seu formato. Os métodos de diagramas espirais, que assumem a função de conversores da árvore em $B D D$, requerem que não haja redundâncias na árvore; isto é, que ela esteja isenta de cortes não-mínimos. Neste artigo sugere-se, uma série de métodos de remoção de redundâncias de árvores de falhas, a princípio coerentes, inicialmente inspirada no método de redução de Faunet.
\end{abstract}

Palavras chave: Arvores de Falhas, Redundância, Diagramas Espirais.

\section{Introdução}

O estudo de uma árvore de falhas deduz todas as possíveis combinações de eventos que levam à falha do produto, são os chamados cortes da árvore. Entre os cortes da árvore pode-se separar aqueles que são mínimos; excluindo-se os cortes que contêm outros cortes; ou seja, as redundâncias. Esta exclusão tem o objetivo de resumir a estrutura lógica da árvore aos menores conjuntos de eventos que, se ocorridos, levam ao topo da árvore; isto é, à falha do sistema. O método de redução de Faunet (REAY \& ANDREWS, 2002) esboça a lógica combinatória necessária para a eliminação das redundâncias; apesar de a sua intenção ser reduzir um específico subconjunto da árvore, dadas algumas características. Logo, partindo do método de redução de Faunet, é proposto, neste artigo, uma série de métodos capazes de remover todas as redundâncias da árvore, com o principal objetivo de permitir a aplicação posterior dos diagramas espirais (FIRMINO et al., 2004). Naturalmente, uma vez removidas todas as redundâncias, qualquer dos métodos de análise de árvores de falhas é favorecido, uma vez que será necessário apenas discriminar os cortes diretamente; porém, o direcionamento para diagramas espirais deriva da capacidade de conversão, da árvore para o formato BDD, dos seus métodos.

\section{Definições}

Para melhor compreensão do que é tratado neste artigo, segue alguns termos que serão bastante usados:

Conexão(ramificação): ligação de um evento básico, ou subsistema, a outro evento básico ou subsistema. 
Subsistema: cada porta lógica da árvore de falhas constitue um subsistema, cujos elementos são as suas ramificações, sejam eles eventos básicos ou outros subsistemas.

Conector (topo): evento básico, ou subsistema, mais próximo da raíz da árvore de falhas, em relação àquele que é uma ramificação sua.

Diagrama Espiral: grafo cujas arestas (conexões ou ramificações) são direcionados para os topos.

\section{Subsistemas}

Como foi dito anteriormente, o que define quais serão os subsistemas são as portas lógicas presentes na árvore de falhas. Observando a figura 1, os subsistemas $G_{1}$ e $G_{2}$ comporiam os dois mais comlpexos subsistemas da árvore de falhas; os quais poderiam ser compostos por outros subsistemas, e assim por diante. Enfim, como os subsistemas formam a árvore de falhas, o topo é, também, um subsistema, com a particularidade de não ser uma ramificação de um outro subsistema.

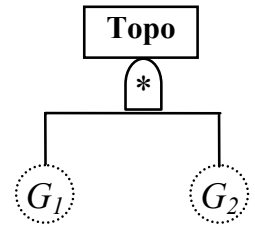

(a)

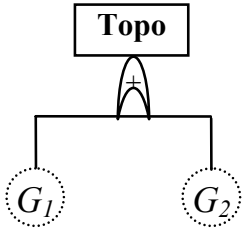

(b)

Figura 1- Subsistemas de uma árvore de falhas.

\section{Incoerência, Inconsistência e Redundância}

Os cortes inconsistentes são provenientes de árvores de falhas inconsistentes, mal construídas às vezes pela falta de conhecimento sobre o sistema ou pela falta de experiência do profissional responsável por sua construção. Em geral, a inconsistência se apresenta quando existem subsistemas compostos por eventos básicos que representam a não-falha dos seus componentes. Neste trabalho, têm sido tratados apenas os casos em que não se verifica a presença de inconsistência; já que são tratadas somente árvores coerentes.

De acordo com Jung et al. (2004), considerando a função booleana, da árvore, representada de forma linear para qualquer evento básico $x_{i}, f_{x_{i}}=a \cdot \bar{x}_{i}+b x_{i}+c$, onde $a, b$ e $c$, são funções booleanas das outras variáveis conectadas a $x_{i}$ e $\bar{x}_{i}$ é o evento complementar a $x_{i}$, a árvore de falhas é dita coerente se a função booleana $a$ é sempre vazia; isto é, $a$ pode ser descartada, e é este tipo de árvore de falhas que está sendo tratado neste artigo. Com isso, $f_{x_{i}}=b x_{i}+c$.

A redundância (ou repetição desnecessária) de eventos básicos é mais freqüente em grandes árvores de falhas. Torna-se cada vez mais difícil evitá-la, à medida que o nível de detalhamento do sistema aumenta.

Considerando como evento original da redundância, aquele mais próximo do topo da ocorrência da redundância (a raíz da qual o evento original e o redundante são descendentes) e considerando como eventos redundantes aqueles mais distantes do topo da ocorrência da redundância, são estudados quatro tipos de ocorrência da redundância:

1. O evento original e os redundantes pertencem à primeira geração do topo da ocorrência da redundância. Nesse caso a solução para a redundância é simples, basta excluir todas as redundâncias e manter o evento original; pois, em álgebra booleana, $A+B+A=A+B$. 
Considerando, na figura 1 , que $G_{1}$ e $G_{2}$ são o mesmo evento básico, este tipo de ocorrência de redundância se verifica.

2. Apenas o evento original pertence à primeira geração do topo da ocorrência da redundância. Aquí ocorrem duas lógicas de matemática combinatória. Se os topos dos eventos redundantes possuirem portas lógicas diferentes da do topo do evento original (que é também o topo da ocorrência da redundância), o topo das redundâncias deve ser eliminado da árvore; observe a figura 2. Porém, Se os topos dos eventos redundantes possuirem portas lógicas iguais à do topo do evento original, apenas os eventos redundantes devem ser eliminados dos seus topos. Observe a figura 3. É importante que se comente que, dada a ocorrência da redundância e a sua resolução, $G_{l}$ passa a possuir apenas uma ramificação e deve ser excluído da árvore. Sua ramificação deve passar a ser uma ramificação do seu topo. Veja que a árvore descrita na figura 2 também passa por este processo. Firmino et al., 2004 detalham este e outros tratamentos.

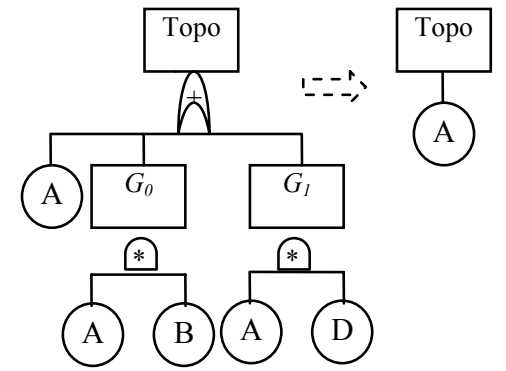

(a)

(b)

Figura 2- Ocorrência de redundâncias, onde apenas o evento original pertence à primeira geracão do tono da ocorrência da redundância.

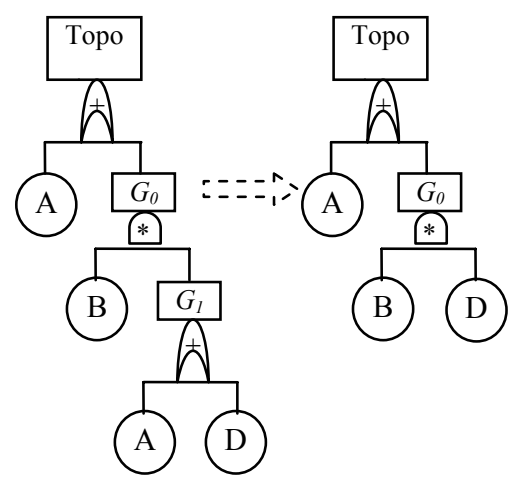

(a)

(b)

Figura 3- Ocorrência de redundâncias, onde apenas o evento original pertence à primeira geração do topo da ocorrência da redundância.

Este tipo de redução utiliza-se do seguinte fato: Seja $f$ a função booleana referente à árvore de falhas apresentada na figura 3(a), em álgebra booleana, $f=A+B \cdot(A+D)=A+B \cdot D$; e, em se tratando da árvore de falhas referente à figura 2(a), $f=A+A \cdot B+A \cdot D=A$.

3. Tanto o evento orginal, quanto os eventos redundantes, pertencem à segunda geração de ramificações do topo da ocorrência da redundância. É neste tipo de ocorrência de redundância que o método de redução de Faunet (REAY \& ANDREWS, 2002) se aplica. Utilizando álgebra booleana, $(A+B) \cdot(A+C)=A+B \cdot C$; assim como, $A \cdot B+A \cdot C=A \cdot(B+C)$; onde, do lado direito destas duas igualdades a redundância é eliminada, como pode ser visto na figura 4. 


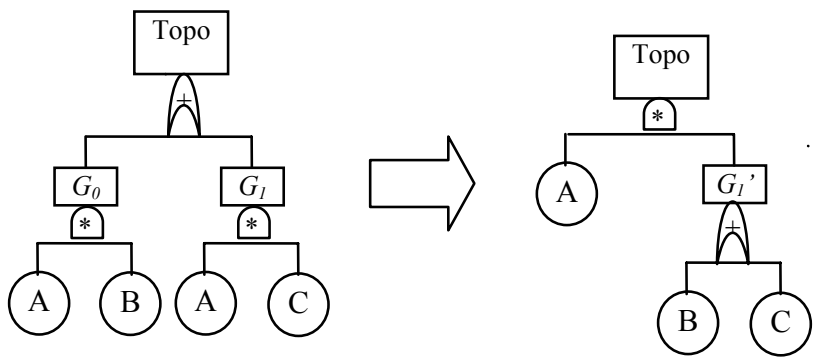

Figura 4- Ocorrência de redundâncias, onde tanto o evento orginal, quanto os eventos redundantes, pertencem à segunda geração de ramificações do topo da ocorrência da redundância.

4. Não há qualquer das relações de parentesco citadas anteriormente e a porta lógica do topo da ocorrência da redundância é do tipo E. Este tipo de ocorrência de redundância é o que requer mais esforço computacional, para ser solucionado, de todos os tipos apresentados. O método de resolução utilizado é o método espiral de eliminação de cortes não-mínimos (MEEC), proposto por este artigo. O MEEC utiliza-se, do fato de que $\left(G_{i}+G_{j}\right) \quad\left(G_{k}+G_{l}\right)=G_{i} \quad\left(G_{k}+G_{l}\right)+G_{j} \quad\left(G_{k}+G_{l}\right) \quad[1]$

onde $G_{i}, G_{j}, G_{k}, G_{l}$ são subsistemas da árvore de falhas. Considerando que a primeira parcela do lado direito da igualdade não contém o evento original, e que, conseqüentemente, a segunda parcela o contém, esta lógica de igualdade é aplicada recursivamente na segunda parcela, até que o evento original seja uma ramificação do, então, topo da ocorrência da redundância; quando isto acontece é possível aplicar a lógica de solução para o caso em que apenas o evento original pertence à primeira geração do topo da ocorrência da redundância, chegando-se, assim, à solução possível. Em seguida, aplica-se a lógica inversa à primeira parcela do lado direito da igualdade de [1], correspondente á última chamada da recursividade. Esta lógica inversa remove o topo do evento repetido da primeira parcela do lado direito da igualdade de [1], quando apenas o evento repetido é removido da segunda parcela, e remove apenas o evento repetido da primeira parcela do lado direito de [1], quando o topo do evento repetido é removido da segunda parcela.

Existe um específico caso em que a definição de evento original e a aplicação da lógica inversa são alteradas. Quando o evento redundante pertencer à terceira geração do topo da ocorrência da redundância, este deve passar a ser considerado como o evento original, a não ser que o evento original também possua esta característica. Além disso, quando o evento original possuir a característica de pertencer à terceira geração do topo da ocorrência da redundância, a lógica inversa não deve ser aplicada à primeira parcela do lado direito de [1], mantendo-a inauterada.

Para o tipo de ocorrência de redundância onde o MEEC se aplica, não é possível que se obtenha uma árvore de falhas sem repetições, mas é garantido que o método de diagramas espirais gere uma OBDD cujos caminhos (cortes) são mínimos e os eventos são otimamente ordenados. O MEEC pode ser melhor compreendido verificando-se a figura 5, onde a sua lógica elimina todos os cortes não-mínimos que, certamente, serão gerados, devido o topo da ocorrência da redundância ser uma porta lógica do tipo E. Como o evento básico B está presente em dois subsistemas distintos e de grau de parentesco elevado (neste caso, o topo da árvore é, também, o topo da ocorrência da redundância e tanto o evento original quanto o evento redundante pertencem à sua terceira geração), apenas o MEEC pode solucioná-lo. Neste caso específico, o evento original poderia ser qualquer uma das ocorrências do evento básico B; porém, quando as ocorrências possuem graus de geração distintos, o evento considerado como original será aquele com o menor grau de geração; ou seja, aquele mais 
próximo do topo da ocorrência da redundância, como já dito anteriormente. O que o MEEC está fazendo de (a) para (b), na figura 5, é considerando que $\left(A+G_{j}\right) \cdot\left(D+G_{l}\right)=A \cdot\left(D+G_{l}\right)+B \cdot C \cdot\left(D+G_{l}\right)$. Como $B \cdot C \cdot\left(D+G_{l}\right)$ é o subsistema que possui a ocorrência da redundância, e como o evento original pertence à primeira geração do topo de tal ocorrência, $G_{l}^{\prime}$; considerando que o evento básico original e o redundante são ramificações de subsistemas cujas portas lógicas são do mesmo tipo, apenas o evento básico redundante deve ser excluído do seu topo, em $G_{1}{ }_{1}$, e o seu topo não deve ser excluído em em $G^{\prime}{ }_{0}$ [o subsistema $\left.A \cdot\left(D+G_{l}\right)\right]$; já que, neste caso, tanto o evento original quanto o redundante pertencem à terceira geração do topo da ocorrência da redundância; nos demais casos, $G_{l}$ seria excluído. Observando a figura 5(c), percebe-se que, agora, os eventos básicos D, E e o prórpio $\mathrm{B}$ possuem uma repetição. Estas repetições são inevitáveis, devido às características da árvore proposta; porém, são necessárias para que seja garantida a geração de todos os cortes mínimos. Como os seus topos são subsistemas cujas portas lógicas são do tipo OU, não haverá problemas com suas permanências na árvore de falhas e suas repetições não devem ser consideradas como redundâncias. O maior problema, ao se tratar de árvores de falhas que possuem esta característica de redundância ou de repetição, é que é impossível que se gere um OBDD mínimo idealmente; ou seja, o OBDD sempre possuirá eventos básicos repetidos.

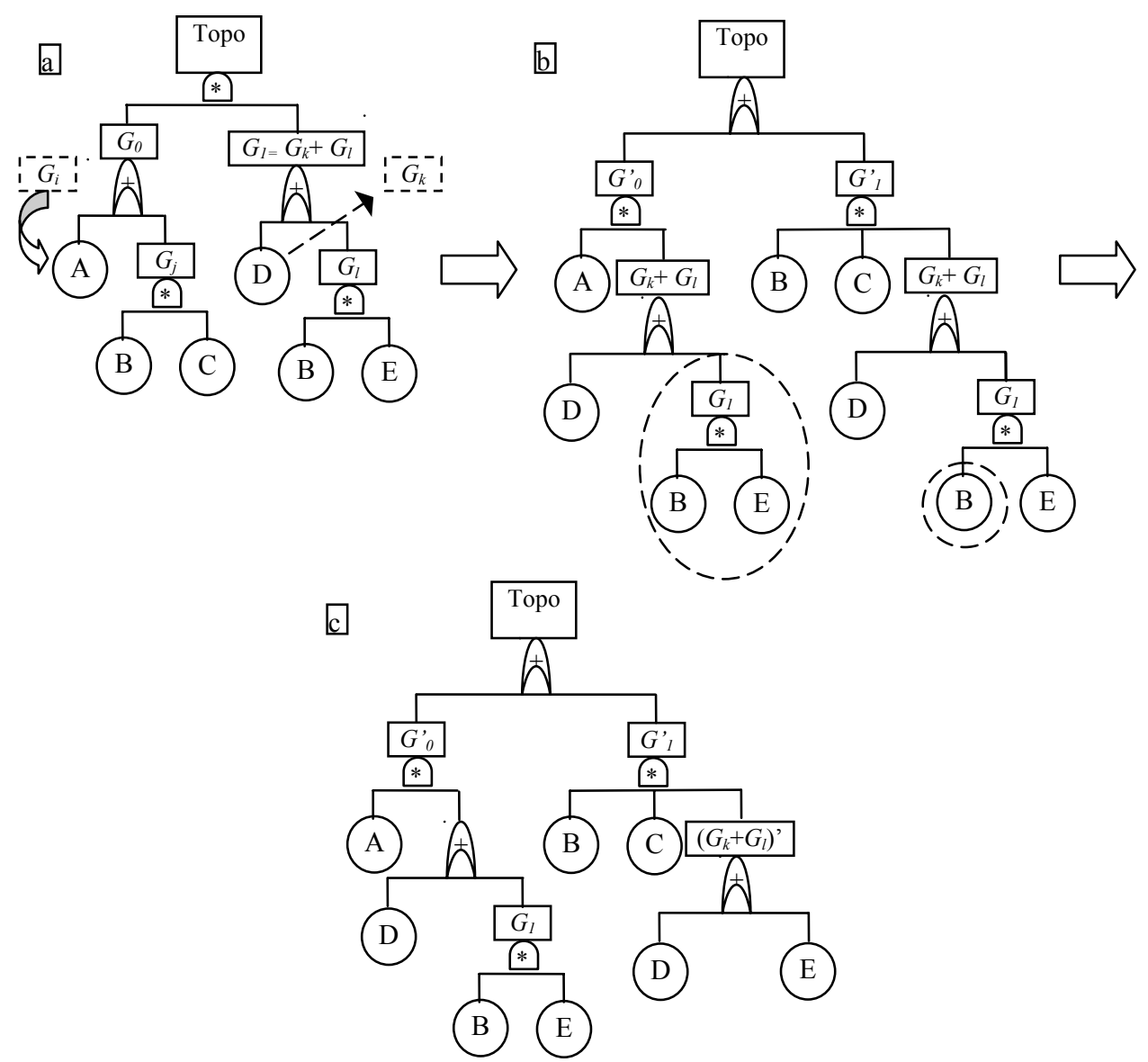

Figura 5- Ocorrência de redundâncias, onde não há qualquer relação de parentesco, evidente, entre o evento original e os redundantes.

Faz-se necessário salientar que apenas após todos os cuidados aquí citados, tanto de redução quanto de reestruturação da árvore de falhas, o método de diagramas espirais pode ser aplicado; caso isto não ocorra, os seus resultados são comprometidos. O método de diagramas espirais, que é responsável direto pela construção do OBDD, requer que a árvore esteja 
ordenada, em relação aos tamanhos dos seus subsistemas, e isenta da presença de redundâncias.

\section{Conclusões}

Com o objetivo de preparar a árvore de falhas para a sua conversão para o formato BDD, os métodos de remoção de redundâncias da árvore, contribuem de maneira a não permitir que permaneçam, na árvore, os cortes não-mínimos. Este tratamento anterior à aplicação dos métodos de diagrams espirais garantem que a conversão resultará em um BDD com o menor tamanho possível; isto é, com o número mínimo de variáveis. Por enquanto, tais métodos de remoção de redundâncias, são adequados apenas a árvores coerentes; desafios futuros são aplicá-los a árvores de falhas incoerentes.

\section{Referências}

FIRMINO, P. R.; MOREIRA, P. I. \& DROGUETT, E. L. (2004)- Diagramas espirais: eétodo auxiliar para a resolução ótima de árvores de falhas. Artigo submetido para este congresso.

JUNG, W. S.; HAN, S. H.\& HA J. (2004)- A fast BDD algorithm for large coherent fault trees analysis. Reliability Engineering and System Safety. Vol. 83, p. 369-374.

MOREIRA, P. I.; FIRMINO, P. R. \& DROGUETT, E. L. (2004)- Aplicação do método de diagramas espirais para resolução de árvores de falhas. Artigo submetido para este congresso.

REAY, K. A. \& ANDREWS, J. D. (2002)- A fault tree analysis strategy using binary decision diagrams. Reliability Engineering and System Safety. Vol. 78, p. 45-56. 